\title{
Relationship Between COVID-19 Cases and Vaccination Rates in New York State Counties
}

Halle Cerio, BS | Laura A. Schad, MPH | Telisa M. Stewart, DrPH | Christopher P. Morley, PhD PRiMER. 2021;5:35.

Published: 9/29/2021 | DOI: 10.22454/PRiMER.2021.432215

\section{Abstract}

Introduction: Vaccines against SARS-CoV-2 have been developed with unprecedented speed. The phased introduction of vaccines may be serving to offset the impact of new viral variants and policy relaxation. In order to assess the impact of vaccination, we examined a snapshot of vaccination rates across counties in a single state, at a single time point, comparing them with population-adjusted case counts.

Methods: We calculated descriptive statistics and bivariate correlations for vaccination rates and cases across counties in New York State (NYS). We conducted a linear regression using cases/100K population per NYS county, frozen at a single snapshot in time, as the outcome variable, predicted by percentage of each county's population (completed series/two doses), controlling for county population.

Results: Percentages with one dose and with two doses were highly correlated $(r=.935, P<.001)$ with one another. Both the one dose and two dose $z$ rates were negatively correlated with cases per $100 \mathrm{~K}$ population (not significant). Population size was strongly correlated with cases per 100K $(r=.715, P<.001)$. The two-dose vaccination rate was a significant negative predictor of cases per $100 \mathrm{~K}$ population in NYS counties $(\beta=-.866, P=.031)$, with each percentage point of completed vaccination nearly equating to one case less in the daily count when controlling for county population size $(\beta=2.732, P<.001)$.

Conclusion: While variants may impact vaccine effectiveness, current vaccination efforts are helping forestall some cases in NYS. Widespread vaccination is still an important goal. Primary care providers, public officials, and public health scientists should continue to urgently promote and support vaccination efforts.

\section{Introduction}

The novel 2019 coronavirus disease (COVID-19) caused by the SARS-CoV-2 virus was declared a pandemic by the World Health Organization in March 2020. ${ }^{1}$ The virus is spread primarily through person-to-person contact, ${ }^{2}$ and both airborne transmission and spread by asymptomatic individuals are likely pathways to transmit SARSCoV-2. ${ }^{3,4}$ COVID-19 has been responsible for over 32 million cases and over 572,000 deaths in the United States, numbers which continue to grow each day. ${ }^{4}$

Vaccines against SARS-CoV-2 have been developed with unprecedented speed. Three are presently in use in the United States, including two that use a messenger RNA (mRNA) vector, and one that relies upon an 
adenovirus vector. ${ }^{5-8}$ Based on the limited availability of the supply of COVID-19 vaccine in the United States, the Centers for Disease Control and Prevention (CDC) provided recommendations for phased vaccination, prioritizing health care workers as well as those living and working in long-term care facilities. As supplies increased, eligibility expanded to other frontline essential workers, followed by those over the age of 65 and younger individuals with high-risk comorbidities. ${ }^{10}$ In New York State (NYS), the final groups to become eligible were all other essential workers, followed by healthy adults. ${ }^{10,11}$ All individuals aged 16 years and over subsequently became eligible to receive the vaccine in NYS (April 6, 2021) and across the United States (April $19,2021) .^{12,13}$

In late March 2021, cases of COVID-19 began to rise in NYS and across the United States, representing a third spike in $\mathrm{NYS}^{14}$ and a fourth for the United States as a whole. ${ }^{15} \mathrm{~A}$ combination of new viral variants, ${ }^{16}$ combined with premature relaxation of social distancing measures ${ }^{17}$ that had proved effective earlier in the pandemic, ${ }^{18}$ likely contributed to the latest spike. The phased introduction of vaccines, however, may be serving to offset the impact of new viral variants and policy relaxation. Several reports from the CDC have noted disparities in vaccination coverage by geography and population, noting disparities across the rural-urban divide, ${ }^{19}$ by social vulnerability, ${ }^{20}$ and across county borders. ${ }^{20}$ However, to date, we have seen no studies that directly examine the relationship between vaccination and test positivity rates for COVID-19 at the county level. In order to assess the impact of vaccination, we examined a snapshot of vaccination rates across counties in a single state, at a single time point, comparing them with population-adjusted case counts.

\section{Methods}

The NYS Department of Health shares vaccination rates per county through the NYS COVID-19 Vaccine Tracker tool, using data reported to the NYS Immunization Information System and the New York City Citywide Immunization Registry. ${ }^{21}$ This online resource is updated daily and includes the number of vaccinated individuals per county, including those who have received at least one vaccine dose and those who have received a completed vaccine series. COVID-19 case rates at the county level are also widely available for all states including NYS, via a cases-per-100K population variable based on a 7-day average that is publicly available from Johns Hopkins University. ${ }^{22}$ We extracted NYS data from both sources to calculate the relationship between vaccination and case rates, and to incorporate analysis of county population, as well. We created the data set for analysis using information available on March 31, 2021.

We calculated descriptive statistics and bivariate correlations for vaccination rates (percent of population with one dose, and with two doses or completed series) and cases across the 62 counties of NYS. We then conducted the core analysis as a linear regression, using cases/100K population per NYS county, frozen at a single snapshot in time (March 31), as the outcome variable, predicted by percentage of each county's population (completed series/two doses), controlling for county population. We conducted all analyses using IBM SPSS Statistics $v 27$. As only aggregated public data were used, this project did not constitute human subjects research.

\section{Results}

NYS counties had a mean of 29.7 cases per $100 \mathrm{~K}$ residents (range: 6.2-108.3). On March 31, NYS had vaccinated approximately $31 \%$ of its residents (mean county percent: 30.98 , range: $21.8 \%-57.4 \%$ ) with one dose of vaccine, and a mean of $18 \%$ (mean county percent: 18.12 , range: $12.3 \%-41.8 \%$ ) of residents with a full vaccination course (Table 1$)$.

Percentages with one dose and with two doses were highly correlated $(r=.935, P<.001)$ with one another. Both the one-dose and two-dose rates were negatively correlated with cases per $100 \mathrm{~K}$ population, but the 
association was not significant at the .05 level in bivariate testing. Population size was strongly correlated with cases per $100 \mathrm{~K}(r=.715, P<.001)$. Further detail is provided in Table 2.

The two-dose vaccination rate was a significant negative predictor of cases per 100K population in NYS Counties $(\beta=-.866, P=.031)$, with each percentage point of completed vaccination nearly equating to one case less in the daily count on March 31, when controlling for county population size $(\beta=2.732, P<.001$; Table 3$)$.

\section{Conclusion}

While variants of concern (B.1.1.7/Alpha, P.1/Gamma, B.1.351/Beta, B.1.167.2/Delta) may impact vaccine effectiveness, current vaccination efforts appear to have nonetheless helped forestall some cases in New York State during spring 2021. Despite any concerns about vaccine effectiveness in the face of emerging variants, widespread vaccination, with those vaccines that are currently available, is still an important goal. Population levels, and possibly by implication, population density, are also related to case emergence. Large gatherings and business openings may still be risky. This underscores the need to offset derestriction with robust vaccination efforts.

This study faced several limitations. First, data series of vaccination rates were not readily available, and many data relevant to COVID-19 were difficult to acquire. This study was performed with a snapshot of 1 day of data. Nevertheless, we strongly believe a time series would reveal the same information. There are also reporting errors or discrepancies as data are reported from counties, to states, and then aggregated in public reporting mechanisms. We believe these data are robust enough to be used in the analysis as presented. Also, the role of COVID-19 variants was not considered in this study. Public data on variant tracking are extremely limited, both by limited reporting and by the existence of good variant tracking itself. It is also important to note that, as a cross-sectional, single-day, retrospective, observational study of association, we cannot establish a causal link between vaccination rates and test positivity per county with this analysis. It is, however, highly suggestive of the importance of vaccination.

Limitations aside, we believe this study provides additional evidence for the effectiveness of the current vaccines available in the United States in stemming the tide of emergent COVID-19 infections. Even though the Delta variant appears to evade both natural and vaccine-induced immunity to some extent, current vaccines in the United States are still effective at preventing serious illness and hospitalization. ${ }^{23,24}$ Future research is already underway across the world assessing the effectiveness of vaccines against new variants, and we urge that such research continue across the spectrum, from the serological to the population level. Meanwhile, primary care providers, public officials, and public health scientists should continue to urgently promote and support vaccination efforts.

\section{Tables and Figures}

Table 1: Rates of COVID-19 Cases per 100,000 Residents, and Rates of Vaccination, Calculated for New York State Counties ( $N=62)$ on March 31, 2021

\begin{tabular}{|l|c|c|c|}
\hline & Minimum & Maximum & Mean (SD) \\
\hline Cases/100K & 6.20 & 108.30 & $29.66(21.3862)$ \\
\hline Population \%: one dose & $21.76 \%$ & $57.42 \%$ & $30.98 \%(5.76 \%)$ \\
\hline Population \%: two doses & $12.27 \%$ & $41.79 \%$ & $18.12 \%(4.81 \%)$ \\
\hline
\end{tabular}


Table 2: Pearson Correlation Coefficients $(P)$ for 62 New York State Counties

\begin{tabular}{|l|c|c|c|c|}
\hline & Pct 1 Dose & Pct 2 Dose & Population & Cases/100K \\
\hline Population \%: one dose & 1.00 & $0.935(P<.001)$ & $-0.108(P=.404)$ & $-0.226(P=.078)$ \\
\hline Population \%: two doses & $0.935(P<.001)$ & 1.00 & $-0.096(P=.458)$ & $-0.096(P=.458)$ \\
\hline Total population & $-0.108(P=.404)$ & $-0.096(P=0.458)$ & 1.00 & $0.715(P<.001)$ \\
\hline Cases/100K & $-0.226(P=.078)$ & $-0.096(P=0.458)$ & $0.715(P<.001)$ & 1.00 \\
\hline
\end{tabular}

Table 3: Estimation of Effect of Rate of Full Vaccination on COVID-19 Cases per 100k County Population in New York State on March 31, 2021, Controlling for Population Size, Via Ordinary Least Squares Regression

\begin{tabular}{|l|c|c|c|}
\hline \multicolumn{1}{|c|}{ Variable } & $\boldsymbol{\beta}$ & $\mathbf{t}(\boldsymbol{P})$ & $\mathbf{C l}$ \\
\hline (Constant) & 36.74 & $4.90(<.001)$ & $21.72-51.75$ \\
\hline Population \%: two doses & -0.87 & $-2.21(.031)$ & $-1.65--0.08$ \\
\hline Population (100K) & 2.73 & $7.92(<.001)$ & $2.04-3.42$ \\
\hline
\end{tabular}

\section{Acknowledgments}

Conflict Disclosure: The authors listed have no relationships, circumstances, or conditions that present a potential conflict of interest.

\section{Corresponding Author}

Christopher P. Morley, PhD

Department of Public Health \& Preventive Medicine,Upstate Medical University, 750 E Adams St, Weiskotten Hall 2262, Syracuse, NY 13210. 315-464-1520

morleycp@upstate.edu

\section{Author Affiliations}

Halle Cerio, BS - Department of Public Health \& Preventive Medicine, State University of New York Upstate Medical University, Syracuse, NY

Laura A. Schad, MPH - 1Department of Public Health \& Preventive Medicine, State University of New York Upstate Medical University, Syracuse, NY

Telisa M. Stewart, DrPH - Department of Public Health \& Preventive Medicine, Department of Urology, and Department of Geriatrics, State University of New York Upstate Medical University, Syracuse, NY Christopher P. Morley, PhD - Department of Public Health \& Preventive Medicine, Department of Family Medicine, and Department of Psychiatry \& Behavioral Sciences | State University of New York Upstate Medical University, Syracuse, NY

\section{References}

1. Guan WJ, Ni ZY, Hu Y, et al; China Medical Treatment Expert Group for COVID-19. Clinical characteristics of coronavirus disease 2019 in China. N Engl J Med. 2020;382(18):1708-1720. 
doi:10.1056/NEJMoa2002032

2. How COVID-19 Spreads. Centers for Disease Control and Prevention. Updated July 14, 2021. Accessed September 23, 2021. https://www.cdc.gov/coronavirus/2019-ncov/prevent-getting-sick/how-covidspreads.html

3. Johansson MA, Quandelacy TM, Kada S, et al. SARS-CoV-2 Transmission from people without COVID-19 symptoms. JAMA Netw Open. 2021;4(1):e2035057. doi:10.1001/jamanetworkopen.2020.35057

4. United States: COVID-19 Overview. Johns Hopkins Coronavirus Resource Center. Accessed September 23, 2021. https://coronavirus.jhu.edu/

5. COVID-19 Vaccination. Centers for Disease Control and Prevention. Page Reviewed August 11, 2021. Accessed September 23, 2021. https://www.cdc.gov/vaccines/covid-19/index.html

6. Developing COVID-19 Vaccines. Centers for Disease Control and Prevention. Updated September 8 , 2021. Accessed September 23, 2021. https://www.cdc.gov/coronavirus/2019-ncov/vaccines/distributing /steps-ensure-safety.html

7. Keech C, Albert G, Cho I, et al. Phase 1-2 Trial of a SARS-CoV-2 Recombinant spike protein nanoparticle vaccine. N Engl J Med. 2020;383(24):2320-2332. doi:10.1056/NEJMoa2026920

8. Johnson \& Johnson's Janssen COVID-19 Vaccine Overview and Safety. Centers for Disease Control and Prevention. Updated June 23, 2021. Accessed September 23, 2021. https://www.cdc.gov/coronavirus /2019-ncov/vaccines/different-vaccines/janssen.html

9. CDC's COVID-19 Vaccine Rollout Recommendations. Centers for Disease Control and Prevention. Updated August 30, 2021. Accessed September 23, 2021. https://www.cdc.gov/coronavirus/2019ncov/vaccines/recommendations-process.html

10. New York State's COVID-19 Vaccination Program. New York State Department of Health. Accessed September 23, 2021. https://covid19vaccine.health.ny.gov/

11. Herd immunity and COVID-19 (coronavirus): What you need to know. Mayo Clinic. August 28, 2021. Accessed September 23, 2021. https://www.mayoclinic.org/diseases-conditions/coronavirus/indepth/herd-immunity-and-coronavirus/art-20486808

12. Governor Cuomo Announces New Yorkers 30 Years of Age and Older Will Be Eligible to Receive COVID-19 Vaccine. Office of the Governer of the State of New York. https://www.governor.ny.gov /news/governor-cuomo-announces-new-yorkers-30-years-age-and-older-will-be-eligible-receive-covid-19

13. Your COVID-19 Vaccination. Centers for Disease Control and Prevention. September 9, 2021. Accessed September 23, 2021. https://www.cdc.gov/coronavirus/2019-ncov/vaccines/your-vaccination.html

14. Hurley C. Amid surging COVID-19 cases, New York Mayor de Blasio orders 80,000 city workers back to offices. World Socialist Web Site. Published March 28, 2021. Accessed September 23, 2021. https://www.wsws.org/en/articles/2021/03/29/blas-m29.html

15. Baker S, Witherspoon A. US enters a fourth wave as variants drive more coronavirus cases. Axios. Published April 1, 2021. Accessed September 23, 2021. https://www.axios.com/coronavirus-casesinfections-hospital-variants-d0f5850c-6098-431c-b38c-55b82cce2b8c.html

16. Wise J. Covid-19: new coronavirus variant is identified in UK. BMJ. 2020;371:m4857. doi:10.1136/bmj.m4857

17. Tsai AC, Harling G, Reynolds ZC, Gilbert RF, Siedner MJ. COVID-19 transmission in the U.S. Before vs. Before relaxation of state social distancing measures. medRxiv. Published online August 2020:2020.07.15.20154534. doi:10.1101/2020.07.15.20154534

18. Morley CP, Anderson KB, Shaw J, Stewart T, Thomas SJ, Wang D. Social distancing metrics and estimates of SARS-COV-2 transmission rates: associations between mobile telephone data tracking and R. J Public Health Manag Pract. 2020;26(6):606-612. doi:10.1097/PHH.0000000000001240

19. Murthy BP, Sterrett N, Weller D, et al. Disparities in COVID-19 vaccination coverage between urban and rural counties - United States, December 14, 2020-April 10, 2021. MMWR Morb Mortal Wkly Rep. 2021;70(20):759-764. doi:10.15585/mmwr.mm7020e3 
20. Hughes MM, Wang A, Grossman MK, et al. County-level COVID-19 vaccination coverage and social vulnerability - United States, December 14, 2020-March 1, 2021. MMWR Morb Mortal Wkly Rep. 2021;70(12):431-436. doi:10.15585/mmwr.mm7012e1

21. COVID-19 Vaccine Tracker. New York State. Accessed September 23, 2021. https://covid19vaccine.health.ny.gov/covid-19-vaccine-tracker

22. COVID-19 Overview State Overview: New York. Johns Hopkins Coronavirus Research Center. Accessed September 23, 2021. https://coronavirus.jhu.edu/region/us/new-york

23. Bernal JL, Andrews N, Gower C, et al. Effectiveness of COVID-19 Vaccines against the B.1.617.2 (Delta) Variant. 2021;385(7):585-594. https://doi.org/101056/NEJMoa2108891

24. Sheikh A, McMenamin J, Taylor B, Robertson C; Public Health Scotland and the EAVE II Collaborators. SARS-CoV-2 Delta VOC in Scotland: demographics, risk of hospital admission, and vaccine effectiveness. Lancet. 2021;397(10293):2461-2462. doi:10.1016/S0140-6736(21)01358-1

Copyright $@ 2021$ by the Society of Teachers of Family Medicine 